\title{
EVOLUTIONARY ABUNDANCE CHANGES IN THE ENVELOPES OF MODERATE MASS RED SUPERGIANTS
}

\section{(Abstract)}

\author{
U. UUS
}

W. Struve Tartu Astrophysical Observatory, Estonia, U.S.S.R.

In stars with about 3 to $8 M_{\odot}$, undergoing the stage of growth of degenerate carbonoxygen core, the bottom of the convective envelope may descend into nuclear burning region. The more effective the convective heat transport, and the more massive the star, and the lower the heavy element content of the envelope, the more successfully the convection invades into burning shell.

Convection, if penetrating into burning shell, embraces only its upper part. The lower part of burning zone remains always radiative because of the small value of energy flux there. So the mass of the stellar core continues to increase.

When the bottom of convective envelope descends into the region of nuclear transmutations, the chemical composition of envelope begins to change. Soon after the penetration of convection into hydrogen burning shell, the $\mathrm{CN}$-cycle equilibrium ratios are established in the envelope. If convection penetrates deep into burning shell, the carbon to oxygen ratio increases considerably after a preceding steep drop, and these may even be achieved a carbon prevalence over the oxygen. As for the hydrogen content in the envelope, there is not enough time for it to decrease considerably during the $\mathrm{C}-\mathrm{O}$ core growing stage. 